\title{
State Vector: A New Approach to Prediction of the Failure of Brittle Heterogeneous Media and Large Earthquakes
}

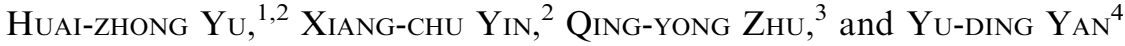

\begin{abstract}
The concept of state vector stems from statistical physics, where it is usually used to describe activity patterns of a physical field in its manner of coarsegrain. In this paper, we propose an approach by which the state vector was applied to describe quantitatively the damage evolution of the brittle heterogeneous systems, and some interesting results are presented, i.e., prior to the macro-fracture of rock specimens and occurrence of a strong earthquake, evolutions of the four relevant scalars time series derived from the state vectors changed anomalously. As retrospective studies, some prominent large earthquakes occurred in the Chinese Mainland (e.g., the M 7.4 Haicheng earthquake on February 4, 1975, and the M 7.8 Tangshan earthquake on July 28, 1976, etc) were investigated. Results show considerable promise that the time-dependent state vectors could serve as a kind of precursor to predict earthquakes.
\end{abstract}

Key words: State vector, acoustic emission, precursor of macro-fracture, earthquake prediction.

\section{Introduction}

The catastrophe of brittle heterogeneous media involves many natural phenomena of which an earthquake may be the most representative example. The physical essence of an earthquake lies in the nonlinear far-from-equilibrium damage process along with the random distribution of initial defects within the source media, which causes the complexity in earthquake prediction. In addition, the information of seismic activities that can be derived from the crust is still relatively limited, which also greatly barricades the prediction of earthquakes. To date, though few earthquakes have ever been successfully predicted globally, considerably more cases

\footnotetext{
${ }^{1}$ State Key Laboratory of Earthquake Dynamics, Institute of Geology, China Earthquake Administration, Beijing 100029, China. E-mail: yuhz@Inm.imech.ac.cn

${ }^{2}$ State Key Laboratory of Nonlinear Mechanics, Institute of Mechanics, Chinese Academy of Sciences, Beijing 100080, China. E-mail: xcyin@public.bta.net.cn

${ }^{3}$ School of Mathematics and Computational Science, Zhongshan University, Guangzhou 510275, China. E-mail: mcszqy@mail.sysu.edu.cn

${ }^{4}$ Guangdong Seismological Bureau, China Earthquake Administration, Guangzhou 510070, China.
} 
were not forecasted. Even though there were dense seismic monitoring networks and highly precise equipment in the regions, the robust precursors of earthquakes still failed to be detected.

Since earthquake prediction is so complicated and difficult, new physical clues or approaches, which reveal the formation and impendence of a large earthquake, should be introduced. Critical point hypothesis (SORneTte and SornetTe, 1990; Sornette and Sammis, 1995; Bowman et al., 1998; Sammis and Smith, 1999; RUNDLE et al., 1999; JAUME and SYKES, 1999) may be an acceptable earthquake prediction model. According to the hypothesis, the earth's crust does not always stay in a critical state. Occurrence of a large earthquake in a region appears to remove the crust from a critical state. Thereafter as the tectonic stress accumulates, the crust will be driven back towards the critical state again. During the establishment of the criticality, seismic moment release accelerates in the region surrounding the epicenter of the ensuing event. The character may be identified by fitting cumulative energy release prior to a large earthquake to a power law time-to-failure relation. Such a fit provides an intermediate-term prediction for a large earthquake. Similar to $\mathrm{CPH}$, the Load/Unload Response Ratio (LURR) method, which narrates the impendence of a large earthquake by anomalous change of LURR, was proposed by YIN et al. (1995; 2000; 2002), whereas, deeper research on the precursors needs to further analyze the damage mechanism of the heterogeneous media in the focal region.

A large earthquake may be considered as a local dynamic rupture process which appears to be a catastrophic transition. A series of recent studies suggest that the process might depend sensitively on the details of heterogeneous source media and stress transfer in the crust (STEIN, 1999). Consequently a possible strategy to identify clues for the prediction of an earthquake is to explore general features of the catastrophic rupture of brittle heterogeneous media. From the viewpoint of damage mechanics, the emergence, accumulation and clustering of micro-cracks will lead to the occurrence of the final failure. The nature of which may quantitatively be described by a special physical approach called state vector, which characterizes the spatial and temporal activity patterns of a physical field (RUNDLE et al., 2002) in the manner of coarsegrain (REICHL, 1980). In recent years, we transplanted the idea of state vector from statistical physics into mechanics to investigate the damage evolution of the brittle heterogeneous systems (YIN et al., 2004a, b).

In this paper, we use both acoustic emission (AE) experiments and seismic data (their catalog retrieved from Center for Analysis and Prediction, China Earthquake Administration) to examine the predictive power of the state vector approach. The experiments were conducted by means of international cooperation. The participants came from State Key Laboratory of Nonlinear Mechanics, Institute of Mechanics, Chinese Academy of Sciences; Center for Analysis and Prediction, Chinese Seismological Bureau; Ioffe Physical Technique Institute, Russian Academy of Sciences; Yunnan Province Seismological Bureau, etc. 


\section{Definition of State Vector}

Figure 1 shows a continuum, which was divided into $n$ sub-regions. The physical quantity $V_{\mathrm{i}}\left(\mathrm{t}_{k}\right)$ (e.g., the sum of Benioff strain) representing the damages in sub-region $i$ within a time window $\mathrm{T}$ can be regarded as $i$-th component of an $n$-dimensional vector $\mathbf{V}_{k}$. This $n$-dimensional vector is called state vector, which slides with a time interval of $\Delta t$. Different state vectors at different times $t_{k}(k=1,2, \ldots)$ can form a trajectory in the phase space. If a series of state vectors at different time steps has been acquired, the temporal and spatial evolution of the physical field may be obtained. To quantitatively measure the evolution of the physical field with state vector, four relevant scalars are defined as follows:

1) The modulus of state vector $\mathbf{V}_{k}$ (represented by $\mathbf{M}$ ), meaning the entire level of the physical field at any time $t_{k}$,

$$
\mathbf{M}=\left|\mathbf{V}_{k}\right|
$$

2) The angle between state vectors $\mathbf{V}_{k}$ and $\mathbf{V}_{k+1}$ (represented by $\varphi$ ), suggesting the change of the physical field between two consecutive times $t_{\mathrm{k}}$ and $t_{\mathrm{k}}+1$,

$$
\varphi=\arccos \left(\frac{\mathbf{V}_{k+1} \cdot \mathbf{V}_{k}}{\mathbf{V}_{k+1} \mathbf{V}_{k}}\right) .
$$

3) The modulus of state vector increment $\mathbf{V}_{k+1}-\mathbf{V}_{k}$ (represented by $\Delta \mathbf{M}$ ), reflecting the change of the physical field between two consecutive times from another viewpoint,

$$
\Delta \mathrm{M}=\left|\mathbf{V}_{k+1}-\mathbf{V}_{k}\right|
$$

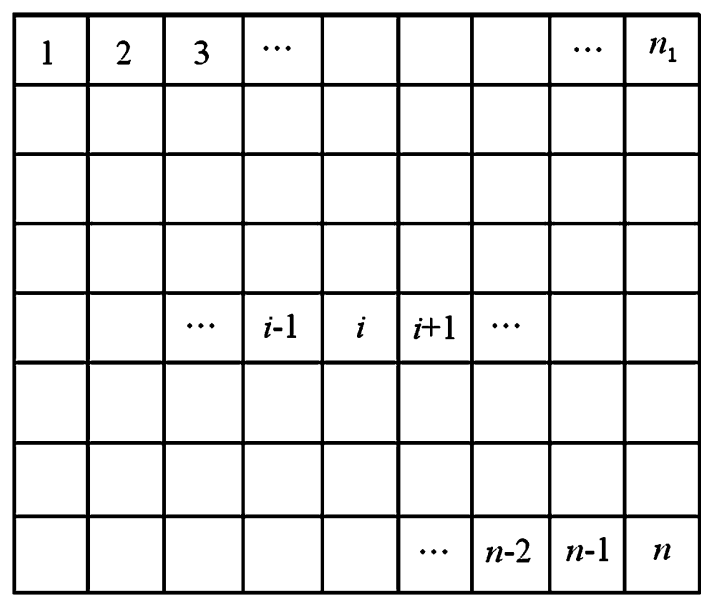

Figure 1

Division of a continuum into $n$ sub-regions. 


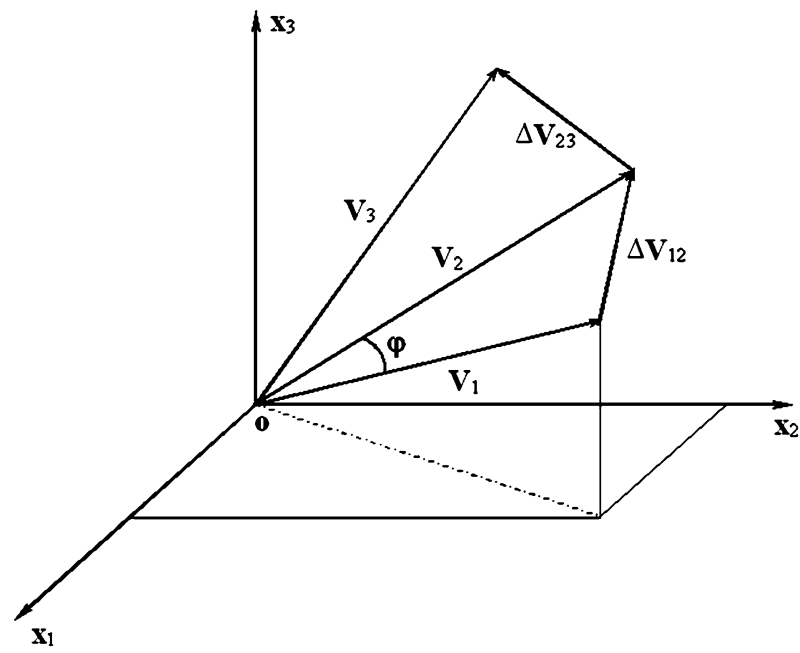

Figure 2

The spatial and temporal patterns of state vectors in three-dimensional phase-space. $\mathbf{V}_{1}, \mathbf{V}_{2}, \mathbf{V}_{3}$ denotes three state vectors at three consecutive times $t_{1}, t_{2}, t_{3} . \varphi$ and $\Delta \mathbf{V}_{12}$ represent respectively the angle and the increment between two consecutive state vectors $\mathbf{V}_{1}$ and $\mathbf{V}_{2}$.

The angle between state vector $\mathbf{V}_{k}$ and equalized vector $\mathbf{V}_{e}$ (represented by $\varphi_{\mathrm{c}}$ ), characterizing the deviation of the physical field from the average field at each time steps,

$$
\varphi_{c}=\arccos \left(\frac{\mathbf{V}_{e} \cdot \mathbf{V}_{k}}{\mathrm{~V}_{e} \mathbf{V}_{k}}\right),
$$

where, the equalized vector $\mathrm{V}_{e}$ consists of equal components.

Figure 2 displays the patterns of state vectors simplified into 3-D phase-space (In practice, more sub-regions are usually provided). It is obvious that with increased damage within the media the value of $\mathrm{M}$ will increase. On the other hand, the value of $\varphi, \varphi_{c}$, and $\Delta \mathrm{M}$ will abruptly increase when the damages within the media begin to cluster.

\section{Experiments Research on State Vector}

Four tri-axial compression acoustic emission (AE) experiments involving two kinds of rocks (Wuding gneiss and Wuding sandstone) were used to research the evolution of the state vectors. Wuding gneiss has a Young's modulus of $\mathrm{E}=27 \mathrm{GPa}$, Poisson ratio $v=0.26$, density $\rho=2.6 \times 10^{3} \mathrm{~kg} / \mathrm{m}^{3}$ and compressional wave speed $\mathrm{c}=3200 \mathrm{~m} / \mathrm{s}$. Wuding sandstone has a Young's modulus of $\mathrm{E}=11 \mathrm{GPa}$, Poisson ratio $v=0.26$, density $\rho=2.2 \times 10^{3} \mathrm{~kg} / \mathrm{m}^{3}$ and compressional wave speed 
$c=2150 \mathrm{~m} / \mathrm{s}$. The geometry of the specimens is $105 \times 40 \times 10 \mathrm{~cm}^{3}$ for gneiss and $105 \times 40 \times 15 \mathrm{~cm}^{3}$ for sandstone. In the experiments, the rock specimens of rectangular prisms were subjected to both lateral and axial loads simultaneously (Fig. 3). Lateral load was symmetrically applied to a specimen with steel plates on the two smaller sides and was retained at a constant pressure of $60 \mathrm{~T}$. Axial load was applied to the specimen with the rams controlled by a hydraulic valve (the maximum of axial load is $500 \mathrm{~T}$ ) until the final fracture took place.

AE signals were recorded continuously with an A-line 32D-AE system made by Ioffe. (Physical Technical Institute, Russian Academy of Sciences and Interunis Ltd.). Each channel consists of an AE sensor, a pre-amplifier and an AECB (Acoustic Emission Channel Board). Ten piezoelectric AE sensors were attached directly to the specimens (small circles represent the AE sensors in Fig. 3). They were used to monitor the sound signal generation from the specimens and convert them into electrical signals that were amplified by a preamplifier and converted into a digital data stream in an AECB (LOCKNER and BYERLEE, 1977). AE features such as arrival times, rise-times, duration, peak amplitude, energy and counts were extracted by a FPGA (Field Programmable Gate Array). The frequency of input filter ranged from $10 \mathrm{kHz}$ to $500 \mathrm{kHz}[-3 \mathrm{~dB}]$ and $24 \mathrm{~dB} /$ octave. The ADC (Analogue to Digital Converter) was operated at a $5 \mathrm{MHz}$ sampling frequency and provided the 16-bit resolution. The threshold values ranged from 45 to $51 \mathrm{~dB}$ and the resolution was $1 \mu \mathrm{V}$. The working frequency of the sensor ranged from 100 to $500 \mathrm{kHz}$ and its

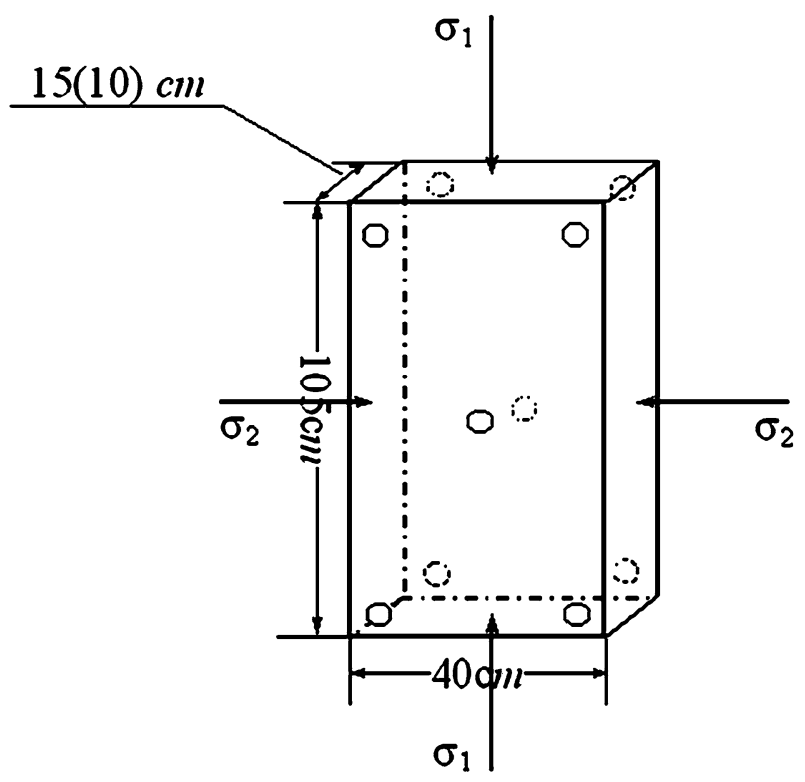

Figure 3

The geometry of rock specimens, the loading conditions, and the arrangement of AE sensors (circles). 
sensitive factor on $175 \mathrm{kHz}$ was $67 \mathrm{~dB}$ relative to $1 \mathrm{~B} / \mathrm{m} / \mathrm{sec}$. AE events were 3-Dpositioned (LOCKNER et al., 1991) by arrival time of AE signals in real time and could be displayed on a screen and recorded for post-processing (YIN et al., 2004a; YU et al., 2005).

For the experiments, the state vector is computed using the sum of AE Benioff strain recorded within each of $n=4 \times 6=24$ sub-regions divided from the larger side of the specimens. Figure $4 \mathrm{a}$ is a plot of the two kinds of axial loads (monotonous and cycling) and the variations of scalars $M, \varphi$ with time for two gneiss specimens ( $\mathrm{G} 1$ and $\mathrm{G} 2$ ), where the unit $\mathbf{J}$ meaning joule. The moving time window $\mathrm{T}$ and sliding

a
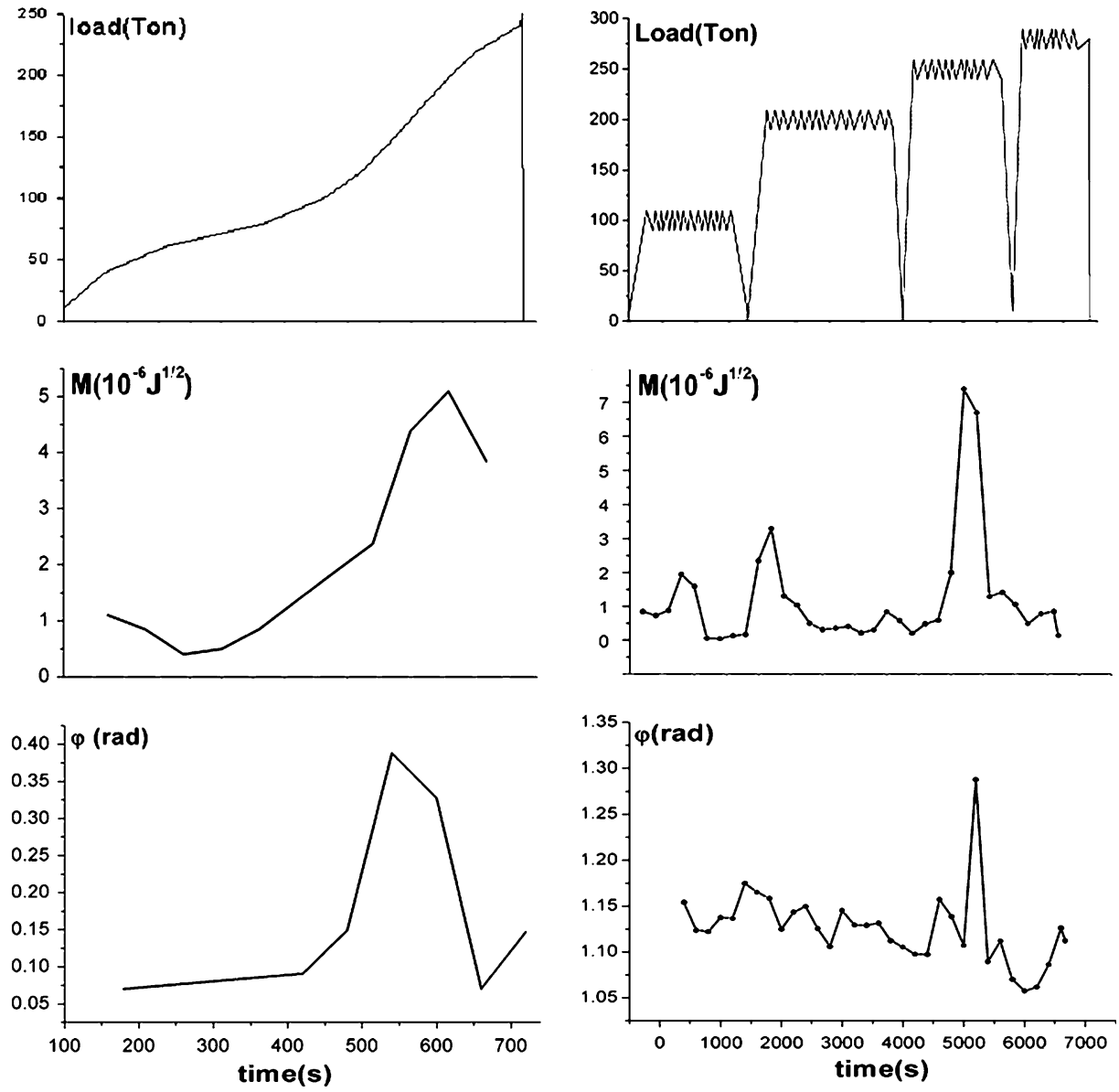

Figure 4

(a) Axial load histories and time series of scalars $M, \varphi$ for two gneiss specimens. Left: G1 and right: G2. (b) Axial load histories and time series of scalars $\mathrm{M}, \varphi$ for two sandstone specimens. Left: S1 and right: S2. 
step $\Delta t$ for G1 was $120 \mathrm{~s}$ and $60 \mathrm{~s}$, along with $T=400 \mathrm{~s}, \Delta t=200 \mathrm{~s}$ for G2. Two sandstone specimens were subjected to the similar load process. Both the axial loads and the evolutions of scalars $\mathrm{M}, \varphi$ time series $(T=150 \mathrm{~s}, \Delta t=100 \mathrm{~s}$ for S1 and $T=$ $400 \mathrm{~s}, \Delta t=200 \mathrm{~s}$ for $\mathrm{S} 2$ ) are shown in Figure 4b. During the first stage of the experiments, there was no coalescence of microcracks within the specimens so scalars $\varphi$ and $\mathrm{M}$ simply fluctuate at a low level. When the microcracks began to coalesce into larger fractures, the damage state of the different sub-regions changed severely as evidenced by sharp peaks in both traces. Following the peak evolution, the macrofracture took place.

b
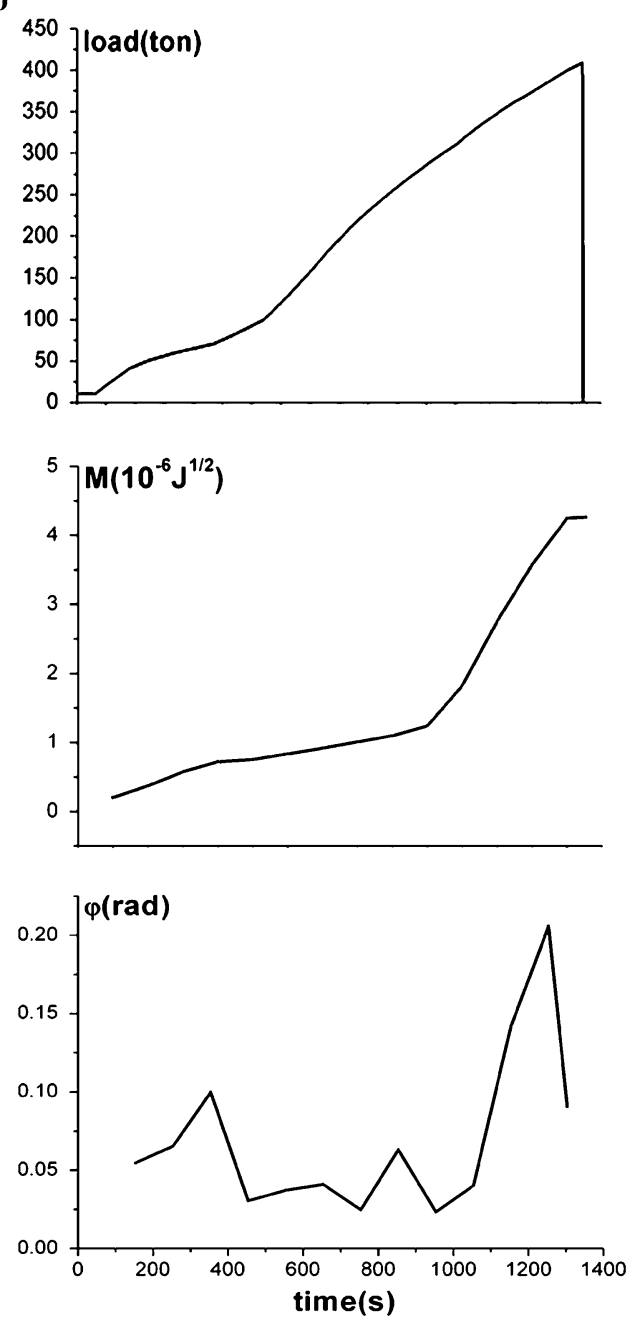
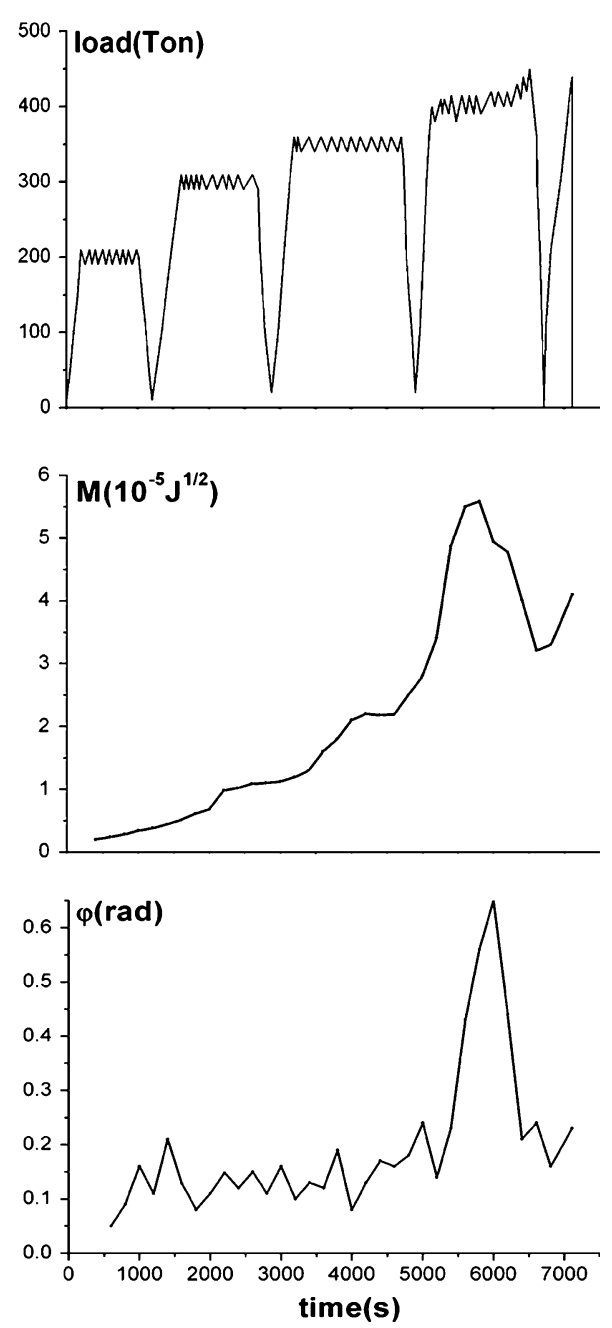

Figure 4

(contd.) 


\section{Application to Seismic Data}

As a retrospective study, we apply the state vector to 'predict' some well known earthquakes which occurred in Chinese Mainland. Figs. 5 and 6 display the evolutions of the four relevant scalars time series before the 1975 Haicheng earthquake and the 1976 Tangshan earthquake, respectively. The spatial windows centered at the epicenter of these two earthquakes $\left(40.7^{\circ} \mathrm{N} ; 122.7^{\circ} \mathrm{E}\right.$ and $39.63^{\circ} \mathrm{N}$; $118.18^{\circ} \mathrm{E}$ ) were taken to be $6^{\circ} \times 6^{\circ}$ and $8^{\circ} \times 8^{\circ}$, and were then divided by the optimal area of $1^{\circ} \times 1^{\circ}$ into 36 and 64 sub-regions. The sums of logarithm of earthquake energy of each earthquake in each sub-region within a period of one year were used to calculate traces of the four scalars by moving them with a time interval of 20 days. Results show that traces of $\mathrm{M}, \Delta \mathrm{M}, \varphi$, and $\varphi_{\mathrm{c}}$ changed anomalously about one month prior to the Haicheng earthquake and three months prior to the Tangshan earthquake. From these two earthquake cases we suggest that the sharp changes of state vectors could be used as medium- or short-term precursors to predict large earthquakes.
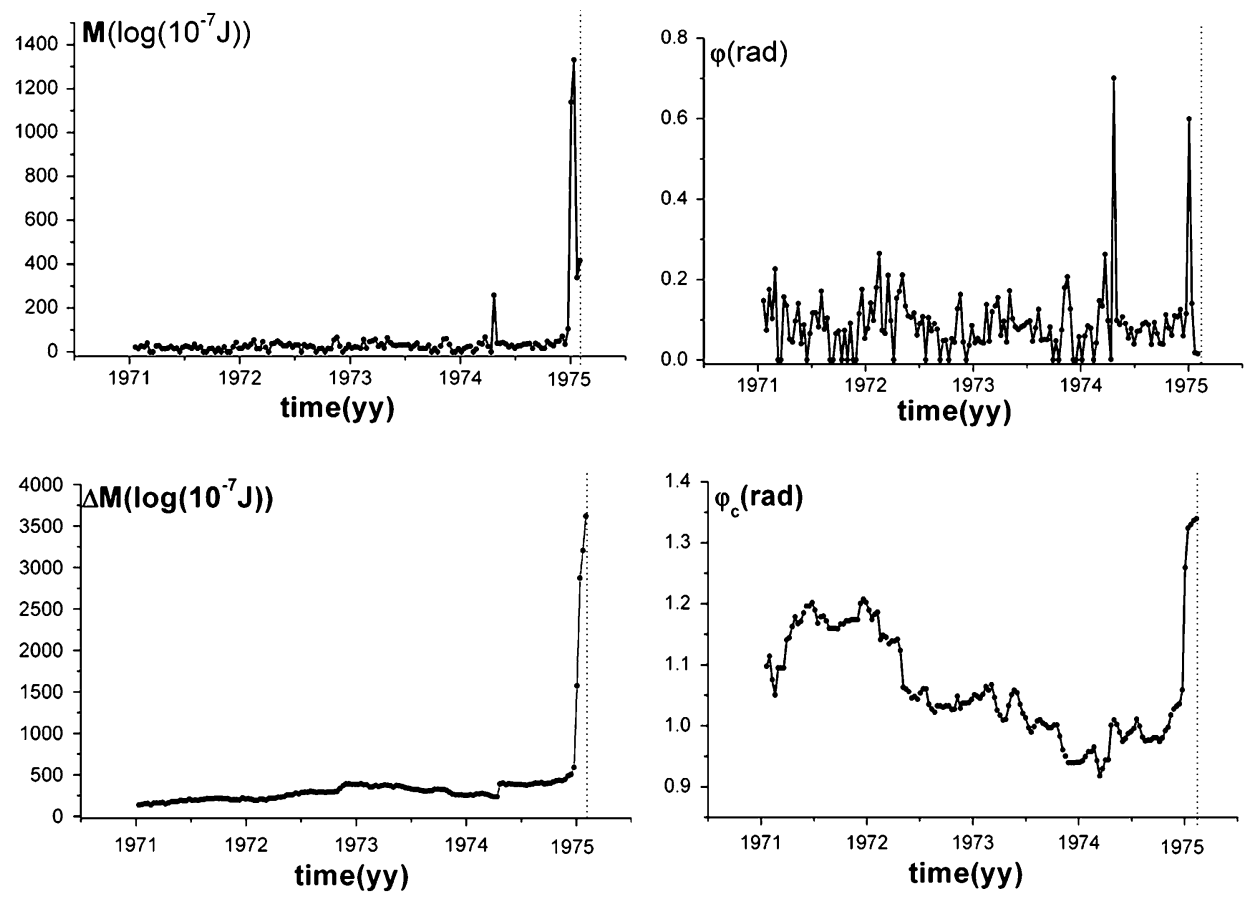

Figure 5

Time series of the four state vector scalars before the M 7.4 Haicheng earthquake. Dashline indicates occurrence of the earthquake. 

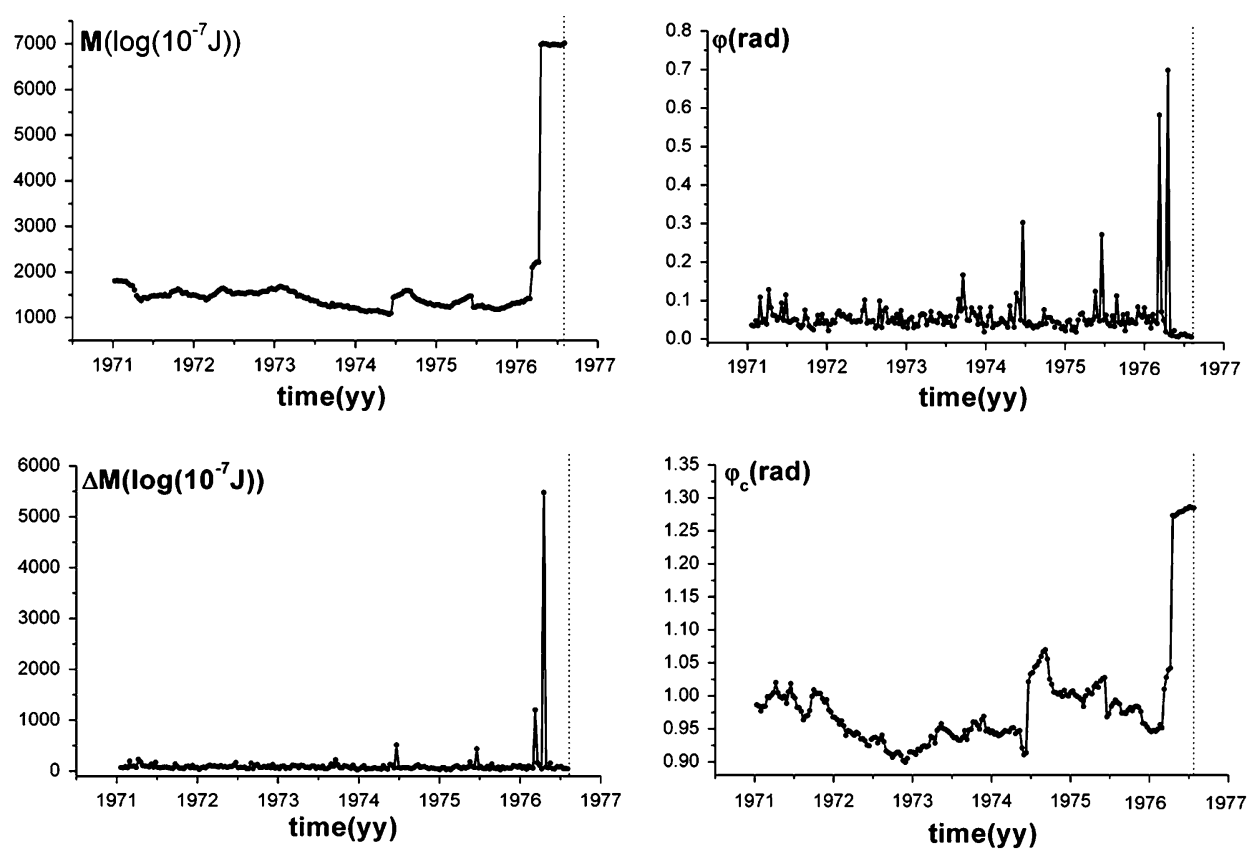

Figure 6

Time series of the four state vector scalars before the M 7.8 Tangshan earthquake. Dashline denotes time of the earthquake.

Figure 7 shows the four relevant scalars evolution time series before two M 6.1 Datong-Yanggao earthquakes. The spatial window centered at the epicenter of the event was $6^{\circ} \times 6^{\circ}$ with a sub-region area of $1^{\circ} \times 1^{\circ}$; and the moving time window was one year at a sliding step of 20 days. It is quite interesting that though the traces did change prior to the first (October 19, 1989) and the second (March 26, 1991) DatongYanggao earthquake, the changes were not as noticeable as that of the Haicheng and Tangshan earthquakes. Furthermore, the variation patterns of state vectors before many moderate $(\mathrm{M} \sim 6)$ earthquakes occurred in the Chinese Mainland, such as the 1976 Helingeer earthquake, and the 1981 Xingtai earthquake, etc. were also studied, but few of these earthquakes could be predicted by the state vector approach.

\section{Predictive Applicability of State Vector}

The fact that the change of state vectors correlates better with the occurrence of larger $(\mathrm{M} \sim 7)$ earthquakes may suggest the predictive power of the approach. To further determine the predictive applicability of the state vector method in earthquake prediction practices, the location precision of earthquakes selected for state vector evaluation should be taken into consideration. Figure 8 shows a selected 

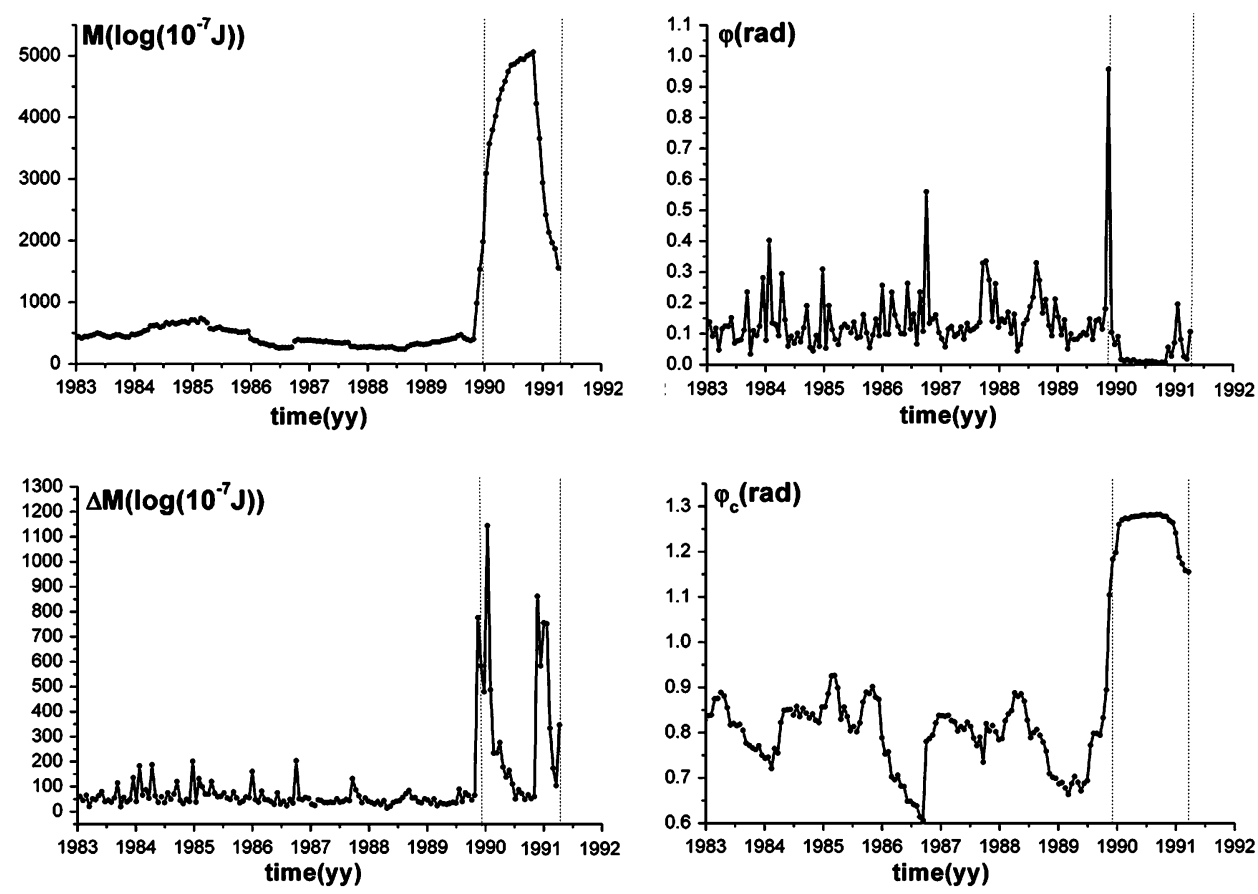

Figure 7

Time series of the four state vector scalars before two M 6.1 Datong-Yanggao earthquakes. Two dashlines represent occurrence of these two earthquakes.

sub-region whose area is determined by the size of length $a$, and the shadow area decided by size $c$ is the precision-dependent area, where the location error impacts the results of the state vector; while in the blank area, the location error will have no effects on the results of the state vector. The higher the location precision of the events is, the smaller the shadow area is, and the less influence will affect the results of the state vector.

If we let

$$
\frac{S_{\text {shadow }}}{S_{\text {total }}}<v,
$$

where $S_{\text {shadow }}$ and $S_{\text {total }}$ represent respectively the area of the shadow region and the entire sub-region, and the ratio $v$ is inverse-correlation with the reliability of the prediction, we can derive

$$
a>\frac{2 c(1+\sqrt{1-v})}{v} .
$$




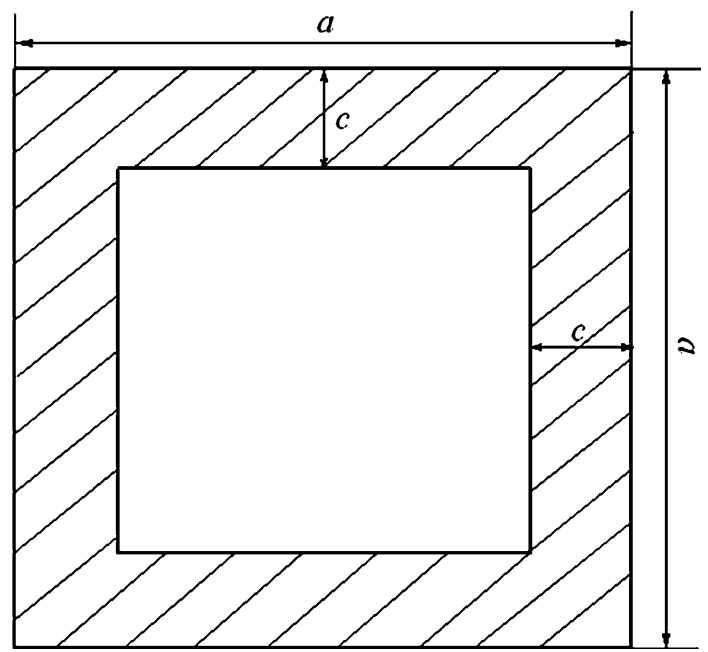

Figure 8

The extent of the location error influence on the state vector. In the shadow area the location error has impacts on the results of the state vector. In the blank area the location error has no effects on the results of the state vector.

On the other hand, the rectangular spatial window selected to compute state vectors is decided by the size of the critical seismogenic region of an earthquake. Thus, the number of sub-regions in a row (e.g., the $n_{1}$ indicated in Fig. 1) should meet

$$
n_{1}<\frac{2 R}{a}
$$

where $R$ is the radius of the critical seismogenic region, and can be defined as

$$
\log (R)=0.087+0.34 \mathrm{M}
$$

where M denotes the magnitude of the earthquake (YIN et al., 2002; 2004a). In earthquake prediction practice, the equation can also be adopted to evaluate the magnitude of an earthquake.

From equations (6) and (7),

$$
R>\frac{n_{1} c(1+\sqrt{1-v})}{v} .
$$

Then combining equation (8) with equation (9), we have

$$
\mathrm{M}>\frac{\log \left(\frac{n_{1} c(1+\sqrt{1-v})}{v}\right)-0.087}{0.34} .
$$


Equation (10) dictates the relationship between the magnitude of the earthquake that can be predicted by state vector and three parameters of $n_{1}, c$ and $v$. Suppose that the earthquake catalog has more precise locations of earthquakes (smaller $c$ ), the smaller earthquakes may be predicted (smaller $\mathrm{M}$ ). However, for a definite precision of earthquake locations, only the larger earthquakes (greater $\mathbf{M}$ ) can be predicted with higher predictive reliability (smaller $v$ ), and also the larger earthquakes (greater M) can be subdivided into more regions (greater $n_{1}$ ) to monitor the heterogeneous damage evolution of the source media.

For the Chinese earthquake catalog $c>5 \mathrm{~km}$, if we let $n_{1} \geq 3$, and $v<0.1$ (the earthquakes could be well evaluated at this level), we can obtain

$$
\mathrm{M}>7.0 \text {. }
$$

The result indicates that with the available Chinese earthquake data, only those earthquakes with magnitude larger than 7.0 could be well predicted by the state vector approach.

\section{Earthquake Prediction Practice}

Figure 9 displays the evolutions of scalars $\mathrm{M}$ and $\Delta \mathrm{M}$ time series in the Yunnan region, China $\left(8^{\circ} \times 8^{\circ}\right.$ centered at $24^{\circ} \mathrm{N} ; 102^{\circ} \mathrm{E}$, optimal sub-region: $1.6^{\circ} \times 1.6^{\circ}$, moving time window: 1 year, and sliding step: 20 days). The anomalous change in evolutions $\mathrm{M}$ and $\Delta \mathrm{M}$ occurred obviously during the period of 2001 to 2004. The results may suggest that a large earthquake with magnitude greater than 7.0 will possibly occur in this region in 1-2 years. According to equations (5) and (10), and
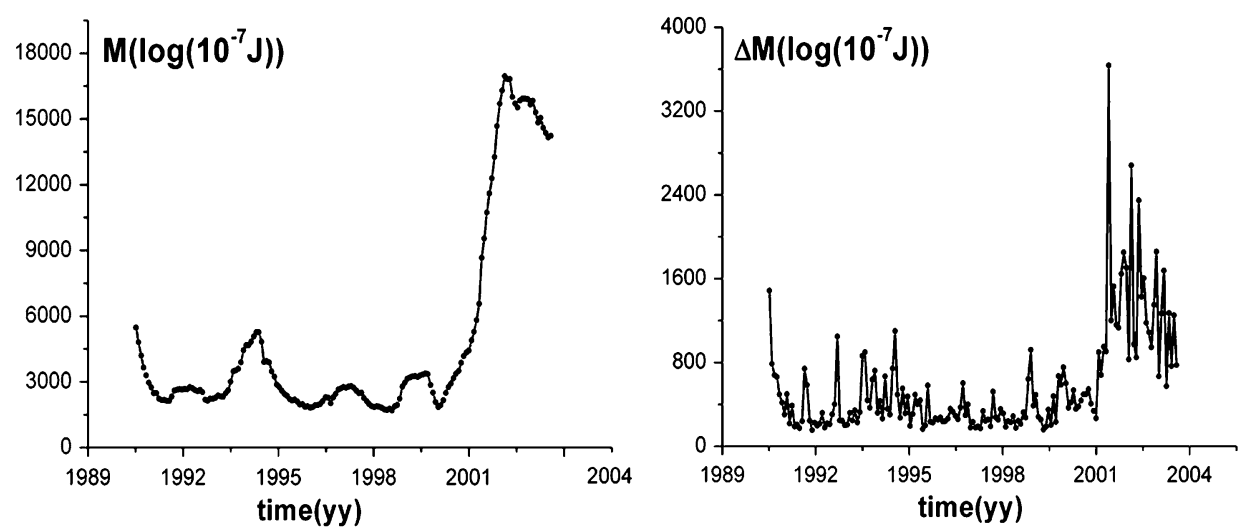

Figure 9

Time series of scalars $\mathrm{M}$ and $\Delta \mathrm{M}$ in the Yunnan district, China. 
the earthquake location precision in this region $(c \approx 10 \mathrm{~km})$, the reliability $(1-v)$ of this prediction is slightly higher than 0.76 , a level with about $50 \%$ probability to properly predict an earthquake from our statistical evaluation (YIN et al., 2004b).

\section{Conclusion}

A new approach to describe damage evolution of brittle heterogeneous media, designated as the state vector, was introduced. Using AE data recorded during rock experiments, it was demonstrated that the anomalous change of state vectors show sharp peaks during the period in which microcracks coalesce into larger fractures, prior to the final failure. Knowing this special character of the state vector, we may use that for testing various kinds of earthquakes. Presently, though the approach is still in its infancy, the attempts in this paper have opened new possibilities to systematically evaluate the hazard potential of future earthquakes. It is doubtless that further progress in the approach will also require more rigorous hypothesis testing and data analyzing (KAGAN and JACKSON, 2001).

\section{Acknowledgments}

The research was supported by the Joint Earthquake Science Foundation of China (Grant No. 606018), China Postdoctoral Science Foundation (Grant No. 2005037384), the National Natural Science Foundation of China (Grant Nos. 40334042, 10232050, 10572154, and 10572140), and special foundation of the Ministry of Science and Technology of China (Grant Nos. 2004CB418403, 2002CB412706 and 2004CB418406).

\section{REFERENCES}

Bowman, D.D., Oulllon, G., Sammis, C.G., Sornette, A., and Sornette, D. (1998), An observational test of critical earthquake concept, J. Geophys. Res. 103, 24359-24372.

JAUme, S.C. and SyKeS, L.R. (1999), Evolution toward a critical point: A review of accelerating Seismic moment/energy release prior to large great earthquakes, Pure Appl. Geophys. 155, 279-306.

KAGAN, Y.Y. and JACKSON, D.D. (2001), Probabilistic forecasting of earthquakes, Geophys. J. Int. 143, $438-453$.

Lockner, D. and ByerleE, J.D. (1977), Hydrofracture in weber sandstone at high confining pressure and differential stress, J. Geophys. Res. 82, 2018-2026.

Lockner, D.A., Byerlee, J.D., and Kuksenko, V. (1991), Quasi-static fault growth and shear fracture energy in granite, Nature 350, 39-42.

ReIchl, L.E., A Modern Course in Statistical Physics (University of Texas 1980).

Rundle, J.B., Klein, W., and Gross, S. (1999), A physical basis for statical patterns in complex earthquake populations: models, predictions and tests, Pure Appl. Geophys. 155, 575-607. 
Rundle, J.B., Tiampo, K.F., Klein, W., and Martins, J.S.S. (2002), Self-organization in leaky threshold systems: the influence of near-mean field dynamics and Its implications for earthquakes, neurobiology, and forecasting, Proc. Natl. Acad. Sci. U. S. A. 99, 2514-2521.

Sammis, C.G. and Smith, S.W. (1999), Seismic cycles and the evolution of stress correlation in cellular automation models of finite fault networks, Pure Appl. Geophys. 155, 307-334.

SORNETTE, A. and SORNETTE, D. (1990), Earthquake rupture as a critical point: consequences for telluric precursors, Tectonophysics 179, 327-334.

SoRnette, D. and SAmmis, C.G. (1995), Complex critical exponents from renormalization group theory of earthquake prediction, J. Phys. I. France 5, 607-619.

STEIN, R.S. (1999), The role of stress transfer in earthquake occurrence, Nature 402, 605-609.

YIN, X.C., Chen, X.Z., Song, Z.P., and YIN, C. (1995), A new approach to earthquake prediction-The Load/Unload Response Ratio (LURR) Theory, Pure Appl. Geophys. 145, 701-715.

Yin, X.C., Wang, Y.C., Peng, K.Y., BaI, Y.L., WAng, H., and Yin, X.F. (2000), Development of a new approach to earthquake prediction: Load/Unload Response Ratio (LURR) Theory, Pure Appl. Geophys. $157,2365-2383$.

Yin X.C., Mora, P., Peng, K.Y., Wang, Y.C., and Weatherly, D. (2002), Load-unload response ratio and accelerating moment/energy release, critical region scaling and earthquake prediction, Pure Appl. Geophys. 159, 2511-2524.

Yin X.C., Yu, H.Z., Kukshenko, V., Xu, Z.Y., Wu, Z.S., Li, M., Peng, K.Y., Elizarov, S., and Li, Q. (2004a), Load-Unload Response Ratio (LURR), Accelerating Moment/Energy Release (AM/ER) and state vector evolution as precursors to failure of rock specimens, Pure Appl. Geophys. 161, 2405-2416.

YIN, X.C., Yu, H.Z., and Zhang, Y.X. (2004b), State Vector - a New method to analysis seismicity, Earthquake Research in China 18, 263-270.

Yu, H.Z., Zhu, Q.Y., YIN, X.C., and WANG, Y.C. (2005), Moment Tensor Analysis of the acoustic emission source in Rock damage process, Progress in Natural Science 15, 609-613.

(Received October 13, 2004, revised September 13, 2005, accepted September 15, 2005)

Published Online First: December 20, 2006

\section{(7) To access this journal online: (4D) http://www.birkhauser.ch}

